\title{
Impact of geometry on flux trapping and the related surface resistance in a superconducting cavity
}

\author{
Felix Kramer®, ${ }^{1, *}$ Oliver Kugeler, ${ }^{1}$ Julia-Marie Köszegi, ${ }^{1, \dagger}$ and Jens Knobloch ${ }^{1,2}$ \\ ${ }^{1}$ Helmholtz Zentrum Berlin, Albert-Einstein-Str. 15, 12489 Berlin, Germany \\ ${ }^{2}$ Universität Siegen, Adolf-Reichwein-Straße 2a, 57076 Siegen, Germany
}

(Received 5 March 2020; accepted 23 November 2020; published 31 December 2020)

\begin{abstract}
In order to minimize the surface resistance in superconducting cavities, a deeper understanding of residual resistance due to trapped magnetic flux is necessary. For that purpose, a combined temperature and magnetic field mapping system is employed to map magnetic flux trapped in a superconducting cavity, and the related increase in surface resistance. By cooling down a $1.3 \mathrm{GHz}$ TESLA single cell cavity several times with externally applied static magnetic fields with different orientations with respect to the cavity, a statement can be made about how the angle between the applied magnetic field and the cavity's surface affects flux trapping, and surface resistance. For example, a significantly higher increase in surface resistance is observed when the applied magnetic field is perpendicular to the cavity's surface compared to when it is parallel.
\end{abstract}

DOI: $10.1103 /$ PhysRevAccelBeams.23.123101

\section{INTRODUCTION}

When operating superconducting cavities, power is dissipated within the cavity wall. Since the cavities are operated at a temperature of around $2 \mathrm{~K}$ the wall plug power needed for $1 \mathrm{~W}$ of dissipated power is close to $1 \mathrm{~kW}$ [1]. For accelerators that are operated in continuous wave mode, reducing of these losses is critical to reduce costs of cryogenic plants.

Losses stem from the nonvanishing surface resistances of superconducting materials in radio frequency (rf) fields. For niobium, which is typically used to fabricate cavities, surface resistance is around $10 \mathrm{n} \Omega$ at $2 \mathrm{~K}$ and for a frequency of the driving rf field of $1.3 \mathrm{GHz}$. The surface resistance consists of two parts: $R_{\mathrm{BCS}}$ and $R_{\text {res }}$. The BCS surface resistance, $R_{\mathrm{BCS}}$, follows from the microscopic theory of superconductors as formulated by Bardeen, Cooper, and Schrieffer [2]. It depends on the material used, its treatment as well as the operating temperature, and the frequency of the rf field.

The second part of the surface resistance, residual resistance $R_{\text {res }}$, is not explained by BCS theory. This resistance is temperature independent, and is mainly caused by trapped magnetic flux $[3,4]$.

\footnotetext{
*f.kramer@helmholtz-berlin.de

†Now at Technische Universitt Berlin.
}

Published by the American Physical Society under the terms of the Creative Commons Attribution 4.0 International license. Further distribution of this work must maintain attribution to the author(s) and the published article's title, journal citation, and DOI.
When cooling down a perfect superconductor below transition temperature, all magnetic flux within the superconductor is expelled. This is the Meissner effect. In practice, however, materials contain defects, which pin flux lines, and thus, prevent their expulsion from the superconductor. In $\mathrm{rf}$ fields, these flux lines oscillate causing power to dissipate within the cavity wall which in turn increases the residual resistance [5,6]. Since the residual resistance takes values in the order of several $\mathrm{n} \Omega$, it significantly contributes to the overall surface resistance if the cavity is operated at $2 \mathrm{~K}$ and $1.3 \mathrm{GHz}$.

With increasing quality standards for superconducting cavities, losses from trapped flux must be considered to push cavities to their limits. Since complete shielding of the earths and other stray magnetic fields is impossible, it is critical to understand how magnetic flux lines are trapped, and how they increase surface resistance. We use a combined temperature and magnetic field mapping system. This setup enables us to map the temperature of the outer cavity wall of a $1.3 \mathrm{GHz}$ TESLA single cell cavity, and the magnetic field surrounding the cavity.

With the experiment presented here, we intend to study how the amount and orientation of trapped flux depends on the orientation of an externally applied magnetic field during cooldown. Additionally, the corresponding surface resistance is investigated. The cavity is cycled through its transition temperature with externally applied static magnetic fields in different orientations. The amount and orientation of trapped flux is then examined with magnetic field sensors while the surface resistance is investigated by means of temperature maps and quality factor measurements. 
In subsequent sections, we present data from magnetic field sensors suggesting that a simple static model cannot sufficiently describe the magnetic flux density trapped within the cavity wall. An increase of the residual resistance is observed when the field is applied parallel to the cavity axis opposed to when the field is applied perpendicularly to it. Furthermore, temperature maps are used to calculate the local surface resistance caused by trapped flux. Here, we observe the largest increase in surface resistance where the trapped flux is perpendicular to the cavity's surface.

\section{EXPERIMENT}

\section{A. Setup}

Measurements are conducted on a $1.3 \mathrm{GHz}$ TESLA single cell cavity fabricated from fine grain niobium $(\mathrm{Nb}$ with RRR 230; $130 \mu \mathrm{m} \mathrm{BCP;} 800^{\circ} \mathrm{C} ; 100+50 \mu \mathrm{m} \mathrm{EP}$; $\mathrm{HPR}$; bake at $139^{\circ} \mathrm{C}$ for $12 \mathrm{~h}$ [7]). All experiments are carried out in a vertical test stand with double magnetic shielding to reduce background magnetic flux density to less than $1 \mu \mathrm{T}$. In addition to the rf diagnostics measuring the quality factor, a combined temperature and magnetic field mapping system is implemented.

The design and initial commissioning of the setup is described in more detail in [8]. A schematic view of the setup is shown in Fig. 1, and Fig. 2 shows a photograph of the setup, partially disassembled. A magnetic field mapping board is mounted on the left hand side, and a temperature mapping board is mounted on the right-hand side of the cavity.

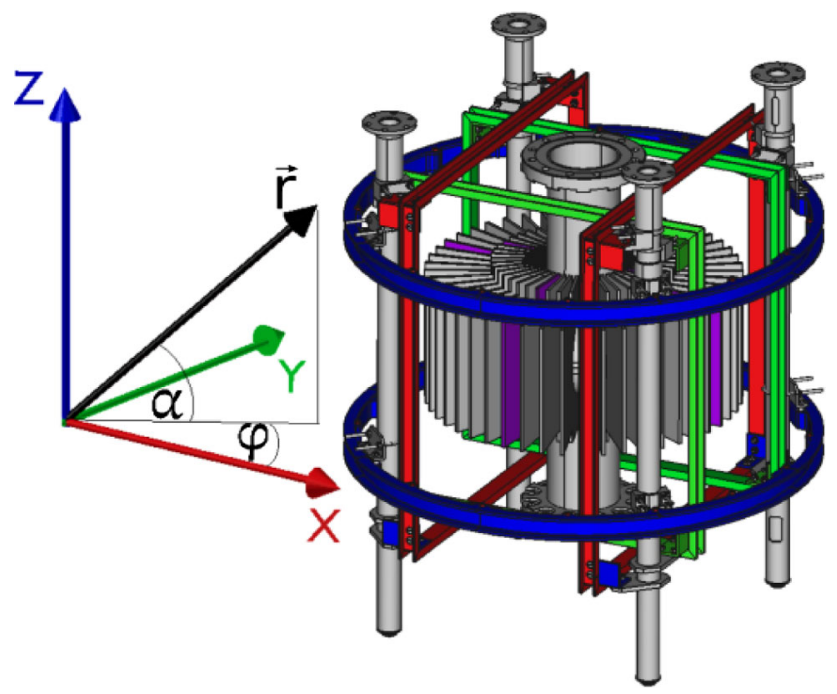

FIG. 1. CAD rendering of the measurement setup consisting of the cavity in the middle, circuit boards measuring the temperature and B-field around it, and three Helmholtz coils. The blue, red, and green coils generate a field in $z, x$, and $y$ directions respectively. Boards for measuring the magnetic field are highlighted purple.
The setup is designed to map the temperature of the cavity wall, as well as the magnetic field surrounding the cavity. To manipulate the surrounding magnetic field, three Helmholtz coil pairs are located around the cavity (see Figs. 1, 2). As a result, the magnetic field can be applied in an arbitrary direction. The coils are optimized to achieve a maximal deviation of the magnetic field of $2 \%$ over the cavity volume [9].

Temperature and magnetic field are mapped using printed circuit boards (PCBs) that are located around the cavity. There are 48 slots around the cavity where the boards can be placed. Four of them are used to install the boards measuring the magnetic field. They are placed at $\varphi=0^{\circ}, 90^{\circ}, 180^{\circ}, 270^{\circ}$. Figure 1 shows the coordinate system used throughout the paper.

A total of 15 magnetic field sensors based on the anisotropic magnetoresistive (AMR) effect, Sensitec AFF755B [10], are installed on each magnetic field measuring board, see [11]. With these sensors, relative measurements with a resolution of $0.02 \mu \mathrm{T}$ are possible. They are placed in five groups of three sensors each (see Fig. 2). For practical reasons, the sensors on the boards are installed in such a way that they measure magnetic field components in a cylindrical coordinate system $(\rho, \theta, z)$. However, throughout the paper only spherical coordinates, as depicted in Fig. 1, are used $(r, \alpha, \varphi)$. The sensors in $\rho$, and $z$ are soldered directly onto the board, whereas, the $\theta$ sensors are connected with angled connectors. The angled connectors are used to rotate the sensors into the correct

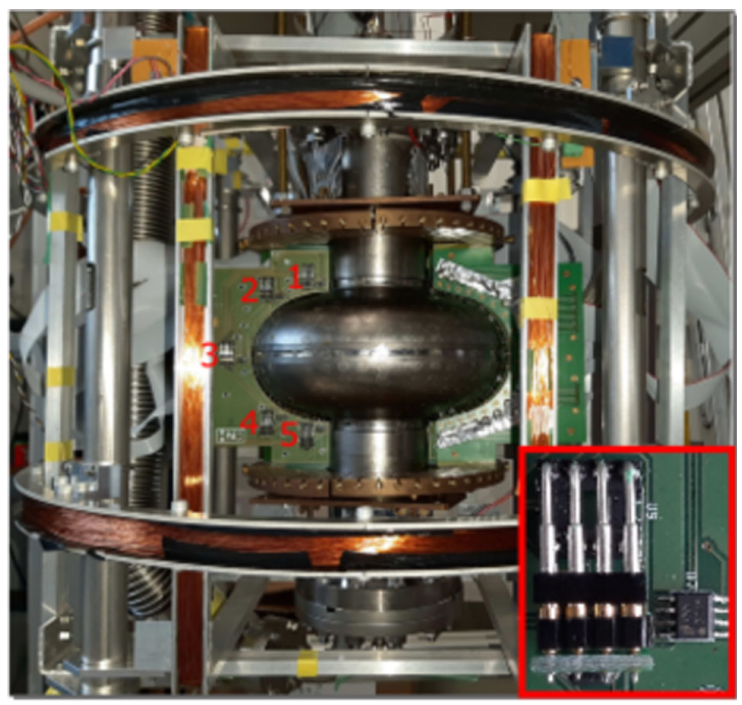

FIG. 2. Setup with cavity, Helmholtz coils, magnetic field mapping card mounted on the left-hand side of the cavity, and temperature mapping card mounted on the right-hand side of the cavity. The AMR sensor groups are labeled from one to five and an enlarged picture of an AMR sensor group is added in the bottom right corner. The sensor measuring in $z$ can be seen on the right, the sensor measuring in $\rho$ is hidden below the adapter of the sensor measuring in $\theta$ which is visible at the very bottom. 
orientation. Using angled connectors widens the board, so one AMR board takes up two of the 48 slots located around the cavity.

The boards for measuring the temperature on the cavity surface were provided by DESY, and are based on the design described in [12]. We use $100 \Omega$ carbon AllenBradley resistors to measure the temperature. Since their resistance is temperature dependent they can be used to measure the temperature by setting a fixed current, and measuring the resulting voltage drop across the resistors. They are pressed against the cavity wall by springs. To achieve good thermal contact Apiezon Type $\mathrm{N}$ grease is applied between sensor and wall to prevent cooling of the active element by the superfluid helium during measurements. Note, Fig. 2 depicts a board with 19 sensors, however, as the cavity gets narrower toward the irises, the sensors would not fit next to each other, so every second board has only 13 sensors. Additionally, Cernox sensors are installed: Two sensors are positioned just below and above the PCBs and are directly mounted to the cavity. An additional sensor measures the helium bath temperature.

\section{B. Measurement procedure}

To obtain a baseline measurement, the cavity is cooled below $9.2 \mathrm{~K}$ without additional applied magnetic field. The standard cooling procedure consists of two steps: A filling step, and a pumping step. During the filling step, the cryostat is filled with liquid Helium at $\mathbf{5 0 0}$ mbar until the cavity is fully immersed. In the subsequent pumping step, pressure is reduced to $16 \mathrm{mbar}$. In this procedure, the liquid Helium level never drops below the top of the cavity. After the baseline measurement, the cavity is warmed up above $9.2 \mathrm{~K}$. For subsequent cooldowns, a magnetic field with flux density of $10 \mu \mathrm{T}$ is applied using the Helmholtz coils. In each cooldown, the orientation of the magnetic field is changed. The polar angle $\alpha$ is changed in steps of $15^{\circ}$ from $0^{\circ}$ to $90^{\circ}$. The azimuthal angle $\varphi$ is changed from $0^{\circ}$ to $90^{\circ}$ while $\alpha$ is $0^{\circ}$. In the different cycles, we attempt to keep the cooling dynamic identical. For each cooldown, the quality factor is measured at different accelerating fields. Additionally, temperature maps, and magnetic field maps are compiled. Magnetic field maps are recorded at three stages during the cooldown: (1) When the cavity is normal conducting, and the Helmholtz coils are turned on, (2) when the cavity is fully superconducting, and the Helmholtz coils are still turned on, and (iii) when the cavity is superconducting, and the Helmholtz coils are turned off. A visualization of the magnetic field surrounding the cavity at all three stages can be found in [13].

\section{MAGNETIC FIELD MEASUREMENTS}

In this section, the question of the orientation, and the amount of trapped flux is investigated. First, the trapped magnetic flux measured after transition to the superconducting state when the Helmholtz coils are turned off, is compared to the results of two simulations. This comparison informs about the amount and orientation of the trapped flux. Second, the magnitude of expelled flux is investigated which provides information about the amount of trapped flux.

Since an absolute calibration is only possible with limited precision, magnetic field data is not presented in absolute terms but in relatives. For calibration, we use the magnetic field value after phase transition to superconductivity with only the background field $(0.5 \pm 0.2) \mu \mathrm{T}$ present. Hence, output voltages during superconductivity and during baseline measurements are assumed to correspond to zero field. Therefore, we measure the additionally trapped magnetic flux once the cavity is superconducting, and the coils are turned off. An example of the measured trapped flux is shown in Fig. 6.

\section{A. Distribution of trapped flux}

The dynamics of partial flux expulsion are difficult to model. Simulating the distribution and orientation of trapped flux in the superconductor under different hypotheses of how the trapping takes place, and by comparing the results to the measured data, we hope to gain insight into the true nature of the trapping mechanism. Since absolute field measurements are only possible with limited precision, a quantitative comparison with simulation results should be viewed with care. Nevertheless, it can give an estimate of how flux is trapped, and how much flux is trapped. In particular, we study whether a simplified static model is able to provide quantitatively accurate results, as is implicitly assumed in a number of publications mentioned further bellow.

COMSOL [14] is used as a simulation tool. Rather than attempting to simulate the trapping mechanism we look at two different final magnetization states as illustrated in Fig. 3. In the first model, we assume a homogeneous B field inside the superconductor, mimicking homogeneous trapping as shown in Fig. 4. In the second model, we assume

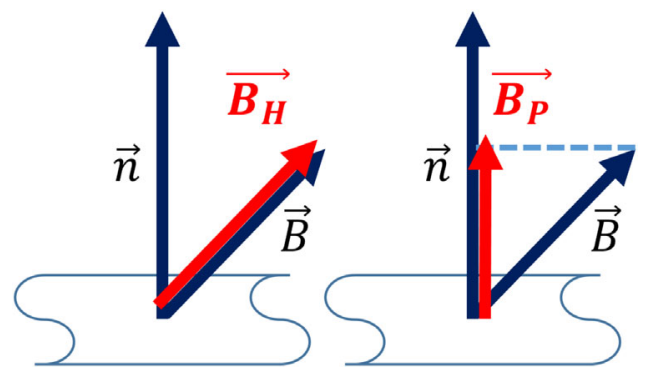

FIG. 3. Sketch of the final magnetization state assumed in the two simulations. $\vec{B}$ is the ambient field, $\vec{n}$ is the normal vector on the cavity surface. The red arrows indicate the assumed trapped field for each model: $\vec{B}_{H}$ for homogeneous trapping same as the externally applied magnetic field, and $\vec{B}_{P}$ for trapping of the field components perpendicular to the cavity surface. 


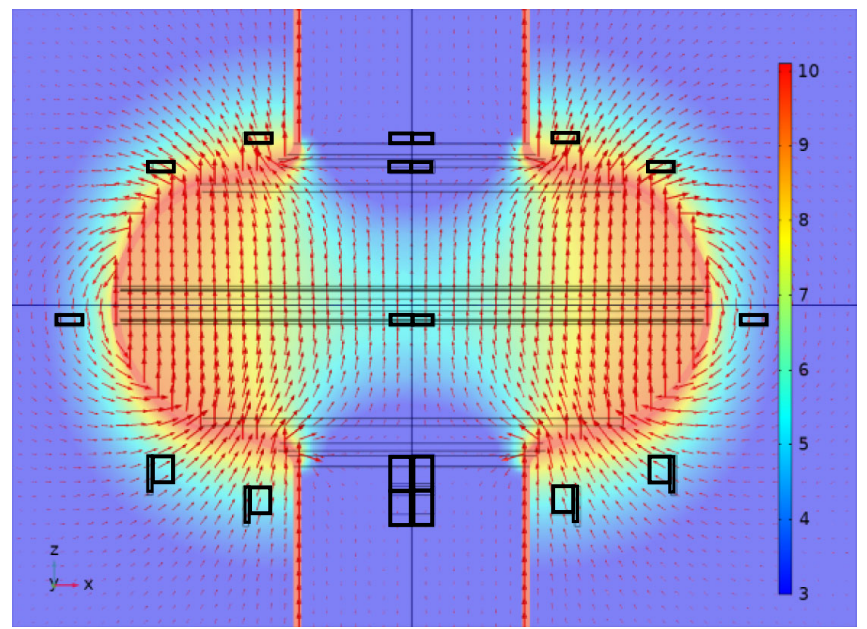

FIG. 4. Simulated slice through the cavity. A homogeneous remanent B-field of $10 \mu \mathrm{T}$ with $\alpha=90^{\circ}$ is set in the cavity walls. Squares envelop the three sensors that belong to a group. Since the individual sensors in the lower two groups are spread further apart, squares are bigger (see Fig. 2).

that only the component perpendicular to the cavity's surface is trapped, the field components parallel to the surface are set to zero (Fig. 5). In the simulations shown here, the field is applied at an angle of $\alpha=90^{\circ}$. With each configuration we can then calculate the field distribution around the cavity using COMSOL, and compare the results to the measured distribution to discern the true behavior of flux trapping. The relative permeability $\mu_{r}$ of the cavity material is set to 0.0001 , so that it represents the cavity in its superconducting state.

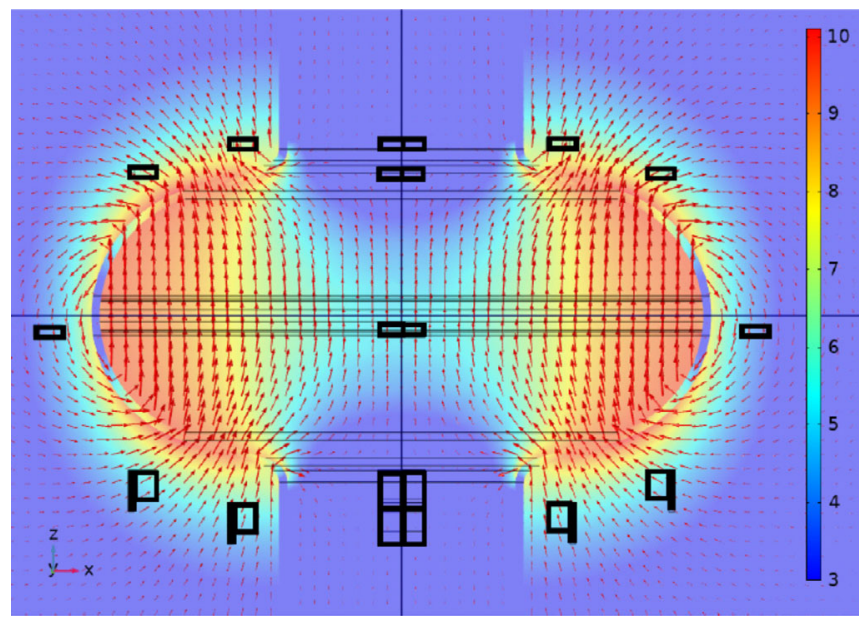

FIG. 5. Simulated slice through the cavity. A remanent B-field of up to $10 \mu \mathrm{T}$ with $\alpha=90^{\circ}$ is set in the cavity walls. Here, only the B-field component perpendicular to the cavity's surface is assumed to be trapped. Squares envelop the three sensors that belong to a group. Since the individual sensors in the lower two groups are spread further apart, squares are bigger (see Fig. 2).

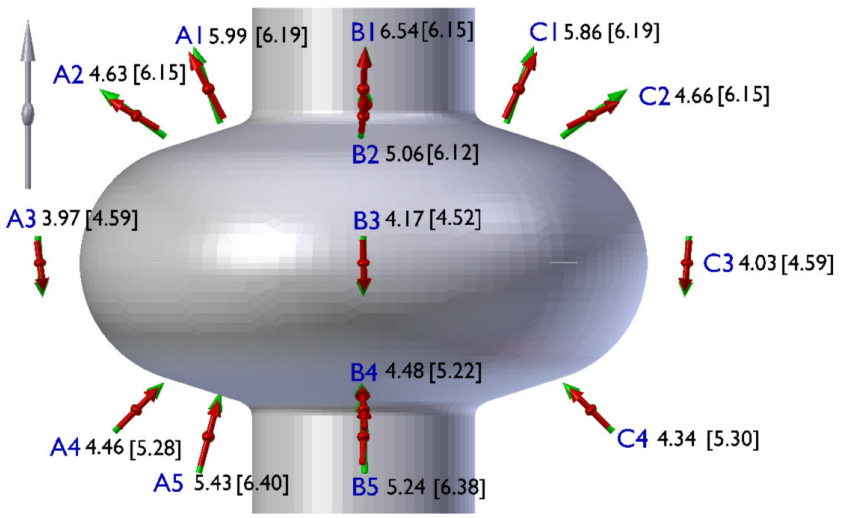

FIG. 6. Measured trapped flux at the sensor positions (red) and simulation results for homogeneous trapping (green). External field is applied in the direction of $\alpha=90^{\circ}$. Numbers next to the arrows show the magnitude of the measured magnetic flux density in $\mu \mathrm{T}$. Numbers in brackets show the magnitude of the simulated magnetic flux density in $\mu \mathrm{T}$. The gray arrow indicates the direction of the applied field, but it is not to scale. The sensor labels are marked in blue. Due to a broken sensor, sensor group C5 is not depicted.

COMSOL uses finite elements to calculate the surrounding magnetic flux density. To check for consistency in the simulations, the applied field is rotated from $\alpha=0^{\circ}, \varphi=0^{\circ}$ to $\alpha=0^{\circ}, \varphi=90^{\circ}$. The root mean square error (RMSE) of the extracted data is $0.03 \mu \mathrm{T}$. Furthermore, the maximum mesh element size is halved increasing the number of domain elements from 605,000 to $10,707,000$. The RMSE of the extracted data is $0.02 \mu \mathrm{T}$. As the deviations are an order of magnitude smaller than the observed differences investigated here, the resolution of the simulations is considered to suffice.

Figure 6 shows a direct comparison of measured data and the simulation results where homogeneous trapping is assumed. To enable a comparison of simulated and measured data, the values from the simulation are evaluated at the actual sensor positions. This accounts for the spread in sensor positions within each sensor group.

In general, the simulation assuming homogeneous trapping agrees well with the data. The magnitude of the measured flux density is smaller than in the simulation which is to be expected since the simulation assumes full trapping, whereas, in the actual cavity flux may have only been partly trapped.

The simulation assuming perpendicular trapped field yields similar results to the simulation assuming homogeneous trapping. As a result, field strength, and field direction from the simulation assuming perpendicular trapping are not visualized because differences to those from the simulations assuming homogeneous trapping are not visually eminent.

To quantitatively compare simulation results, the relative magnetic flux density with respect to the mean magnitude for each data set is calculated for each sensor position. 
TABLE I. Magnetic flux density of measurements, simulation with homogeneous trapping, and simulation with perpendicular trapping normalized to the mean magnitude for each data set, respectively. The external field is applied at $\alpha=90^{\circ}$. If the simulation significantly $(3 \sigma=12 \%)$ differs from the measurements, the value is highlighted in bold. Due to broken sensors, sensor groups C5 and D2 are not included.

\begin{tabular}{lccc}
\hline \hline $\begin{array}{l}\text { Sensor } \\
\text { group }\end{array}$ & Measurement & $\begin{array}{c}\text { Simulation } \\
\text { homogeneous }\end{array}$ & $\begin{array}{c}\text { Simulation } \\
\text { perpendicular }\end{array}$ \\
\hline A1 & 1.21 & 1.19 & 1.14 \\
A2 & 0.93 & $\mathbf{1 . 0 8}$ & $\mathbf{1 . 1 2}$ \\
A3 & 0.80 & 0.83 & 0.84 \\
A4 & 0.90 & 0.87 & 0.90 \\
A5 & 1.09 & 1.06 & 1.05 \\
B1 & 1.32 & $\mathbf{1 . 2 0}$ & $\mathbf{1 . 1 4}$ \\
B2 & 1.02 & 1.09 & 1.12 \\
B3 & 0.84 & 0.83 & 0.84 \\
B4 & 0.90 & 0.86 & 0.89 \\
B5 & 1.06 & 1.06 & 1.05 \\
C1 & 1.18 & 1.20 & 1.14 \\
C2 & 0.94 & $\mathbf{1 . 0 9}$ & $\mathbf{1 . 1 2}$ \\
C3 & 0.81 & 0.83 & 0.84 \\
C4 & 0.87 & 0.86 & 0.89 \\
D1 & 1.35 & $\mathbf{1 . 1 9}$ & $\mathbf{1 . 1 4}$ \\
D3 & 1.06 & $\mathbf{0 . 8 3}$ & $\mathbf{0 . 8 4}$ \\
D4 & 0.74 & $\mathbf{0 . 8 7}$ & $\mathbf{0 . 8 9}$ \\
D5 & 0.97 & 1.06 & 1.05 \\
\hline \hline
\end{tabular}

Thus, the absolute amount of trapped flux is removed from comparison, and only relative differences in trapped flux throughout the cavity factor in. As a result, the quotients for homogeneous flux trapping differ from the ones where nonhomogeneous trapping is assumed. The results from calculating the quotients for $\alpha=90^{\circ}$ are shown in Table I.

But first, we consider the systematic error of the magnetic field measurements by investigating the measured field when the cavity is still normal conducting. Under ideal conditions, the magnetic field should be the same at all points. However, due to imperfectly calibrated sensors, and inhomogeneity in the applied field, the sensors have an error of 4\%. With this knowledge Table I can be interpreted:

Note that identical letters indicate sensor groups of a single card. Identical numbers indicate sensor groups at the same level. It stands out that simulation results differ for specific sensor groups. For example, we see that within both simulations sensor groups on level 1 measure a higher flux density than sensor groups on level 5 . Because in the simulations flux gets trapped uniformly over the cavity, this disparity can only be explained by the fact that the sensors in sensor groups on level 4 or 5 are spread further apart within a group, and therefore, are further away from the cavity wall. Furthermore, simulated values in sensor groups on level 1 differ 5 to 6 percentage points across simulations, whereas, sensor groups on level 3 and 5 differ only 1 percentage point. Since the difference across simulations is larger for sensor groups on level 1 than for sensor groups on level 3 or 5 , we conclude that sensor groups on level 1 are more sensitive to the hypothesis on homogeneous or perpendicular flux trapping than groups on level 3 or 5. Sensitivity differences in sensor groups on level 5 compared to sensor groups on level 1 are caused by the positioning issue mentioned above. Therefore, the sensor position should be adjusted for future experiments.

In Table I, simulation results that differ significantly, i.e., $3 \sigma=12 \%$ from the measurements are highlighted in bold. The card that stands out is D, since the measurements disagree with the simulations in sensor groups D1, D3, and D4. On the other hand, sensors on cards A, B, and C, agree well with simulations and with each other on levels 3-5 or 3-4 in the case of card C. On the upper half of the cavity sensors on card A, and $\mathrm{C}$ as well as card B, and D agree with each other. Sensors on card A and C agree with simulations on level 1 and measure less trapped flux than sensors on B, and D which agree on level 2 with simulations. So, in the upper half we see a symmetry between sensors on cards $\mathrm{A}$, and $\mathrm{C}$ as well as on cards $\mathrm{B}$, and D where each pair disagrees with simulations in one group. To summarize, we see that flux gets trapped differently in the top half than in the bottom half, and we also see that flux gets trapped differently along Card D compared to the others. We also see that for the bold entries the difference between measurement and simulation is larger than the difference between the simulations.

The findings above suggest that a static model poorly reflects reality. In fact, we suspect that the dynamics of flux trapping, i.e., the changing boundary conditions as the superconducting front passes through the cavity, play an important role. Hence, using a static model to describe the entire cavity, might fall short. Therefore, inferences on the extend of flux trapping using only one or a few sensors should treated cautiously. In such cases, only statements about the local environment are permissible. While it is a very useful tool, and was successfully used in [15-17], it should be kept in mind that the real field profile might sometimes be quite different than a static model suggests.

To give an overview of all applied field angles, the RMSEs between simulation and measurements and across simulations are calculated for each applied field angle in Table II.

Again, simulated values $\left(\mathrm{RMSE}_{\text {sim }}\right)$ are closer to each other than to the actual data. This underlines the statements made earlier.

\section{B. Amount of trapped flux}

Apart from the distribution and orientation of trapped flux, we also investigate whether the orientation of the externally applied magnetic field influences the fraction of flux that is trapped. The amount of trapped flux can be calculated in two different ways: by comparing the measurements to the simulations, or by calculating the amount of expelled flux during phase transition. As stated in 
TABLE II. RMSE between measurements and simulation as well as between both simulation models for different applied field angles. The RMSE is calculated using magnetic flux density relative to the mean of all sensors. RMSE $\mathrm{H}_{\mathrm{H}}$ is the RMSE between measurements and simulation assuming homogeneous field, $\mathrm{RMSE}_{\mathrm{P}}$ denotes RMSE between measurement and simulation with perpendicular field, and $\mathrm{RMSE}_{\text {sim }}$ is the RMSE between simulations.

\begin{tabular}{lccc}
\hline \hline$\alpha[\mathrm{deg}]$ & $\mathrm{RMSE}_{\mathrm{H}}$ & $\mathrm{RMSE}_{\mathrm{P}}$ & $\mathrm{RMSE}_{\text {sim }}$ \\
\hline 0 & 0.14 & 0.21 & 0.09 \\
15 & 0.19 & 0.26 & 0.08 \\
30 & 0.31 & 0.36 & 0.06 \\
45 & 0.05 & 0.07 & 0.04 \\
75 & 0.27 & 0.27 & 0.04 \\
90 & 0.1 & 0.12 & 0.03 \\
\hline \hline
\end{tabular}

Sec. III A, this estimation should be treated witch caution but can, nevertheless, be used as an indicator of how much flux is trapped.

\section{Comparison with simulations}

As the simulations assume full trapping, we can estimate how much flux is actually trapped by dividing the magnitude of the measured flux density $\left|\vec{B}_{\text {meas }}\right|$ by the magnitude of the simulated flux density assuming homogeneous trapping $\left|\vec{B}_{\mathrm{H}}\right|$ and the simulated flux density assuming perpendicular trapping $\left|\vec{B}_{\mathrm{P}}\right|$. Comparing the quotients of the measured flux density and simulated flux density in each sensor group is not significantly correlated for trapped flux with different angles $\alpha$. Thus, only averaged quotients for different angles of the applied fields are shown in Table III.

For $\alpha=0^{\circ}$ the fraction of trapped flux is smaller than for $\alpha=90^{\circ}$. This effect is amplified by a systematic error in the magnetic field mapping cards that was only found after measurements completed. As already mentioned in the beginning of this subsection, the accuracy of the data is not

TABLE III. Estimated fraction of trapped flux and its standard variation under the assumption of homogeneous trapping $\left|\vec{B}_{\mathrm{H}}\right|$ and perpendicular trapping $\left|\vec{B}_{\mathrm{P}}\right|$ for different angles of the applied field. The temperature difference of the top and bottom Cernox sensors on the cavity when the lower sensor is at $9.2 \mathrm{~K}$ is quoted in the last column.

\begin{tabular}{lllr}
\hline \hline$\alpha[\operatorname{deg}]$ & $\left\langle\frac{\left|\vec{B}_{\text {maes }}\right|}{\left|\vec{B}_{\mathrm{H}}\right|}\right\rangle[\%]$ & $\left\langle\frac{\left|\vec{B}_{\text {maxa }}\right|}{\left|\vec{B}_{\mathrm{P}}\right|}\right\rangle[\%]$ & $\Delta \mathrm{T}[\mathrm{K}]$ \\
\hline 0 & $63 \pm 2$ & $66 \pm 3$ & 3.107 \\
15 & $51 \pm 3$ & $54 \pm 5$ & 16.152 \\
30 & $73 \pm 10$ & $86 \pm 20$ & 2.607 \\
45 & $72 \pm 1.3$ & $74 \pm 1.2$ & 0.159 \\
75 & $70 \pm 5$ & $72 \pm 5$ & 4.675 \\
90 & $82 \pm 2$ & $86 \pm 3$ & 3.782 \\
\hline \hline
\end{tabular}

sufficient to determine with high precision how much flux is trapped.

\section{Flux expulsion during phase transition}

Another way to quantitatively estimate the amount of trapped flux, is to investigate how much flux is expelled. If a cavity traps all flux, the phase transition cannot be seen by magnetic field probes because the field stays where it is. If the cavity expels all magnetic field generated by the Helmholtz coils, the transition would be seen clearly in the captured data. Since all flux is pushed out of the cavity's volume, flux density would change at the sensors' positions surrounding the cavity. If the B-field is known for, no and complete trapping, it is possible to linearly interpolate between both, and compare the results to the data when flux is partially trapped. Quantifying how much flux is trapped requires three measurement points of the B-field. 1. When the cavity traps all flux, 2. when it expels all the flux, and 3 . when it partially traps flux. The Helmholtz coils are turned on when the field data is taken.

The data points for full and partial trapping are easily obtained for all field configurations. The data point for full trapping is taken by measuring the magnetic field when the cavity is normal conducting. As the field distribution does not change when the cavity traps all flux, this mimics full trapping. The data point for partial trapping is obtained after the cavity is fully superconducting. The data point for complete flux expulsion is, however, more difficult to measure as the cavity always traps some flux. The only way to measure it, is to cool down the cavity through the transition temperature without any field applied, and to apply the field when the cavity is superconducting. In this case, the applied flux cannot enter the cavity wall as it is already superconducting. Thus, the field distribution is the

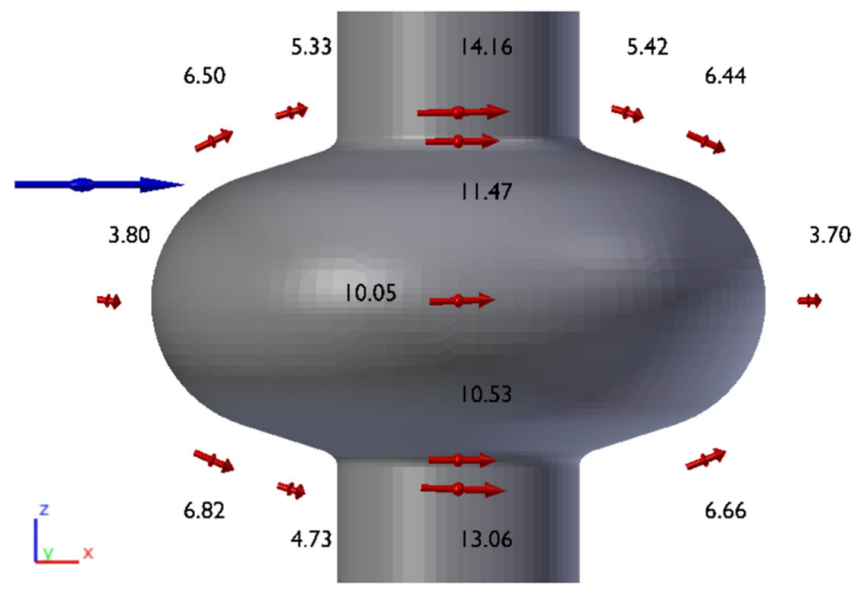

FIG. 7. Measured flux distribution for a field applied in $\varphi=0^{\circ}$ $\left(\alpha=0^{\circ}\right)$ after the cavity is superconducting, this state mimics full expulsion. The numbers next to the arrows show the magnitude of the magnetic flux density in $\mu \mathrm{T}$. The blue arrow indicates the direction of the applied field, but it is not to scale. 
TABLE IV. Estimated trapped flux by analyzing expelled flux during phase transition using Eq. (1).

\begin{tabular}{lc}
\hline \hline$\alpha[\operatorname{deg}]$ & $\langle q\rangle[\%]$ \\
\hline 0 & $81 \pm 2$ \\
90 & $93 \pm 4$ \\
\hline \hline
\end{tabular}

same for a cavity that expelled all applied flux in transitioning to the superconducting state. During the measurements, two data points of this kind are taken. They are retrieved following baseline measurements. Figure 7 shows the magnetic field in this state.

Linear interpolation between both cases, full trapping and full expulsion, leads to:

$$
q=\frac{B_{\mathrm{tf}}-B_{\mathrm{fe}}}{B_{n}-B_{\mathrm{fe}}}
$$

Where $q$ is the fraction of flux trapped, $B_{\mathrm{tf}}$ is the magnitude of the magnetic flux density when flux is partially trapped, $B_{\mathrm{fe}}$ is the magnitude for full expulsion and $B_{\mathrm{n}}$ is the magnitude of mimicked full trapping, i.e., when the cavity is still normal conducting.

The field distribution during phase transition changes perpetually and, therefore, field orientation and magnitude might differ from the expected distribution when it is trapped. Hence, this method gives only an estimate of the amount of flux that is expelled. For instance, in the case of the field applied at $\alpha=90^{\circ}$, some sensors show that more flux is expelled than in the hypothetical case indicating that this model is a simplification of the actual process. Calculating the mean fraction results in Table IV.

\section{INCREASED SURFACE RESISTANCE DUE TO TRAPPED FLUX}

This section discusses the relation between surface resistance and trapped flux. First, we investigate average surface resistance by means of quality factor measurements. Second, local surface resistance is examined by means of temperature maps.

\section{A. Quality factor and average surface resistance}

For each applied field, we conduct quality factor measurements. The quality factor is measured at accelerating fields of 2.5 to $17.5 \mathrm{MV} / \mathrm{m}$ in $2.5 \mathrm{MV} / \mathrm{m}$ intervals at $1.51,1.61,1.72,1.8,1.86 \mathrm{~K}$. Temperature-dependent data can be used to separate BCS and residual surface resistance according to Eq. (2).

$$
R_{\mathrm{s}}=\left(\frac{a f^{2}}{T}\right) \exp \left(-\frac{b T_{\mathrm{c}}}{T}\right)+R_{\text {res }}
$$

Here, $R_{\mathrm{S}}$ is surface resistance, $T_{\mathrm{c}}$ is critical temperature, $T$ is the cavity's temperature, $f$ is the rf frequency, and $a, b$

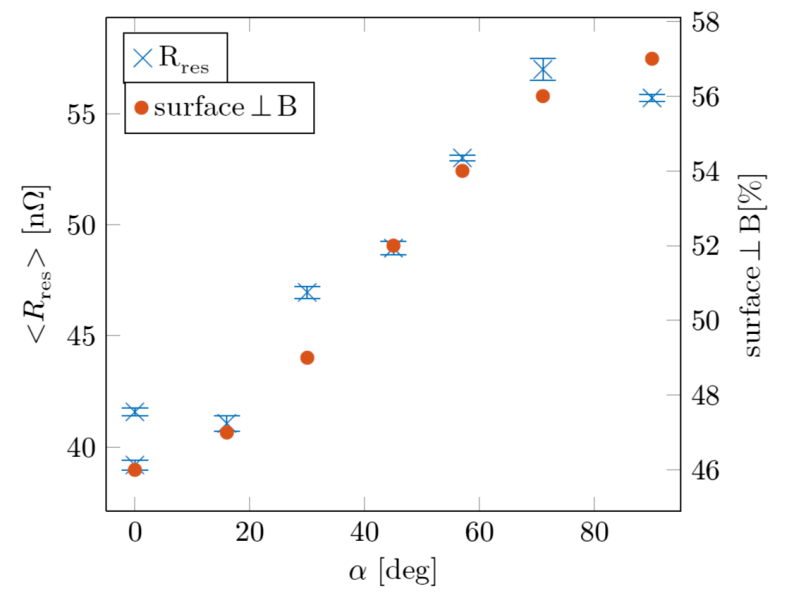

FIG. 8. Measured residual resistance, and percentage of the cavity's surface perpendicular to $\vec{B}$ weighted with $H^{2}$ of the rf field. There are two data points for measured residual resistance at $\alpha=0^{\circ}$ since the field is applied in $\varphi=0^{\circ}$ and $\varphi=90^{\circ}$ with $\alpha=0^{\circ}$. The lower point corresponds to $\varphi=90^{\circ}$.

are fit parameters. Figure 8 depicts the residual resistance at $5 \mathrm{MV} / \mathrm{m}$ versus the polar angle of the applied magnetic field.

As evidenced in Sec. IV B, there are localized hot spots where the field is perpendicular to the surface of the cavity. That raises the question whether only the field perpendicular to the surface contributes to the residual resistance. As the geometry of the cavity is known, we can calculate the fraction $F$ of the surface perpendicular to the B-field by taking the cosine of the angle between the B-field vector $\vec{B}$, and the normal vector $\vec{n}_{i}$ of the surface elements of area $A_{i}$ :

$$
F=\frac{\sum_{i} A_{i} \cdot\left|\cos \left(\measuredangle\left(\vec{B}, \vec{n}_{i}\right)\right)\right|}{\sum_{i} A_{i}} .
$$

Since the square of the $\mathrm{rf}$ magnetic field is responsible for the losses, it must be considered in the calculations. Figure 8 shows residual surface resistance, and the percentage of the surface perpendicular to the magnetic field weighted with $H^{2}$ of the rf field.

The similarity between measurements, and calculated percentages supports the hypothesis that areas where the magnetic field is perpendicular to the cavity's surface have a higher surface resistance than areas where the field is parallel to the surface. This effect has to be considered when conducting experiments on flux trapping.

\section{B. Locally increased surface resistance depending on field orientation}

Notably, hot spots shift dependent on the orientation of the applied magnetic field. This supports the hypothesis that the additional residual resistance is created mainly by field components perpendicular to the cavity's surface. This is consistent with findings in [17]. In order to clarify the 


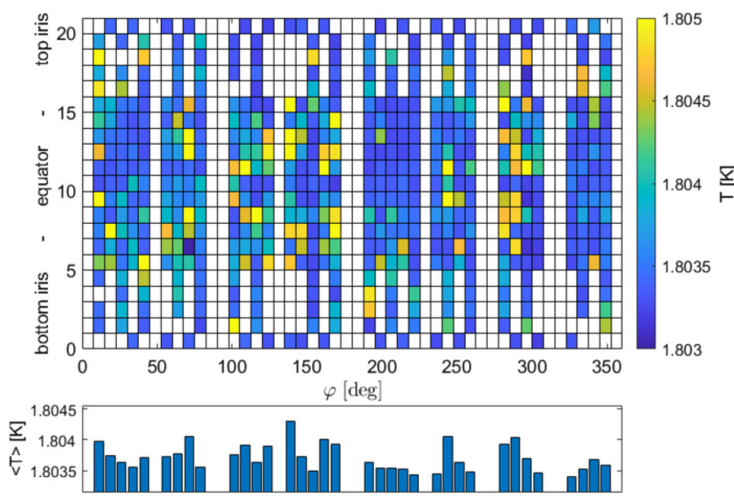

FIG. 9. Temperature map of baseline measurement without any externally applied field. Data is recorded at $1.8 \mathrm{~K}$, and $E_{\text {acc }}=15 \mathrm{MW} / \mathrm{m}$. The quality factor is $Q_{0}=2.7 \times 10^{10}$.

visualization of hot spots, bar charts are added to the bottom, and right-hand side of each temperature map in Figs. 9-12 illustrating average temperatures of each row, or column, respectively. In Figs. 10-12 temperature maps show hot spots.

All temperature maps are recorded at $15 \mathrm{MV} / \mathrm{m}$ and $1.8 \mathrm{~K}$, except for when the field is applied at $\alpha=90^{\circ}$. Due to technical difficulties, measurements with field at $\alpha=90^{\circ}$ are recorded at $1.86 \mathrm{~K}$. The magnetic field is $10 \mu \mathrm{T}$ in all cases, except for zero-field measurements. The temperature maps exhibit several white spots due to lacking data at these positions. This has the following reasons: Two columns are missing at $0^{\circ}, 90^{\circ}, 180^{\circ}$, and $270^{\circ}$ because B-mapping cards are installed at these positions. Other columns are missing because available channels for readout are limited. White spaces toward the irises are due to the narrowing of the cavity limiting sensor space.

The first temperature map in Fig. 9 shows heating without applied field. Throughout the cavity heating is relatively evenly distributed. Heating at the equator is less.

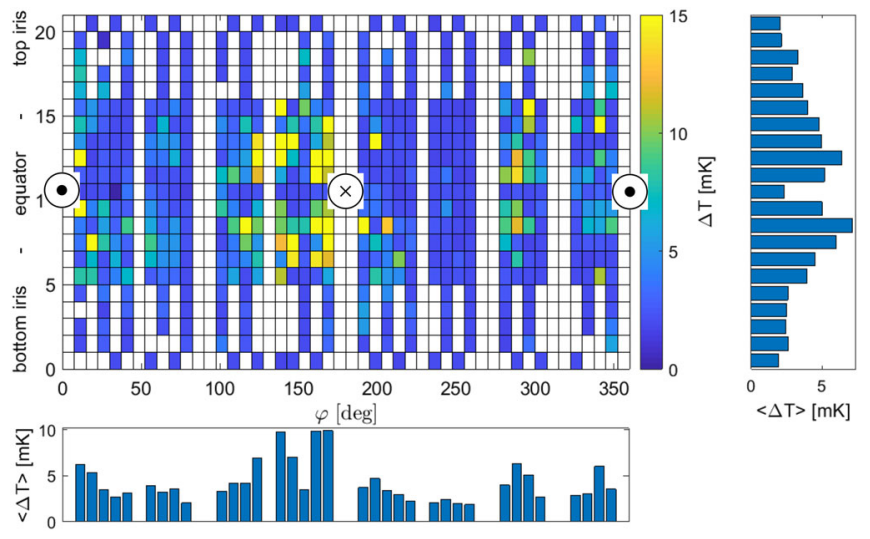

FIG. 10. Relative heating for applied field in $\varphi=0^{\circ}, \alpha=0^{\circ}$. It is indicated where the magnetic field is perpendicular to the cavity's surface. The data is recorded at $1.8 \mathrm{~K}$, and $E_{\text {acc }}=15 \mathrm{MW} / \mathrm{m}$. The quality factor is $Q_{0}=4.8 \times 10^{9}$.

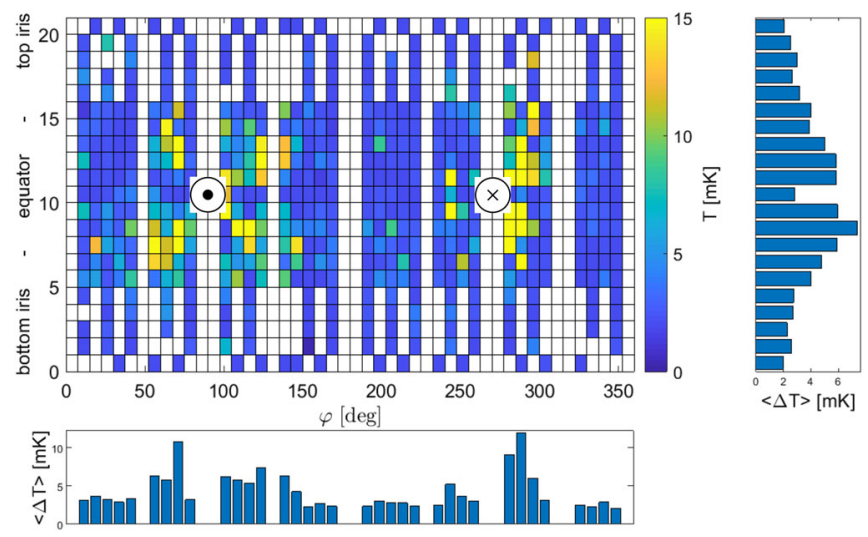

FIG. 11. Relative heating for applied field in $\varphi=90^{\circ}, \alpha=0^{\circ}$. It is indicated where the magnetic field is perpendicular to the cavity's surface. The data is recorded at $1.8 \mathrm{~K}$, and $E_{\text {acc }}=15 \mathrm{MW} / \mathrm{m}$. The quality factor is $Q_{0}=5.1 \times 10^{9}$.

This is probably caused by the weld seam. The contact pressure of the sensors is lower due to the fact that the wall is thinner. Thus, the temperature sensors have poorer contact to the cavity surface.

All subsequent temperature maps show relative heating. The temperature map in Fig. 9 represents baseline measurement and is subtracted from the temperature data of all other field orientations.

In Fig. 10, the field is applied in $\varphi=0^{\circ}, \alpha=0^{\circ}$. In this representation of the temperature map, the field is perpendicular to the equator at $352.5^{\circ}$, and $172.5^{\circ}$. The bar chart for the rows still shows little heating at the equator, presumably due to poor thermal contact explained earlier. But heating increases more where the field is rather perpendicular to the surface. This is more pronounced for the equator than for the irisis where the field is rather parallel. Looking at the columns in Fig. 10, there is a higher variation of temperature across columns in Fig. 10 than compared to columns in Fig. 9. Again, there is increased heating where the field is perpendicular to the surface. This is more obvious by comparing the temperature maps of Figs. 10 and 11.

Figure 11 shows the relative heating when the field is applied at $\varphi=90^{\circ}, \alpha=0^{\circ}$. The magnetic field is now perpendicular to the equator at $82.5^{\circ}$ and $262.5^{\circ}$. We see increasing average temperature across rows similar to Fig. 10, the case of $\varphi=0^{\circ}$. Yet, hot spots rotate $90^{\circ}$ in $\varphi$.

Figure 12 shows the relative heating when the field is applied in $\alpha=90^{\circ}$. Since there were technical issues at the beginning of measuring, there exists no temperature map for $1.8 \mathrm{~K}$ instead measurements are recorded at $1.86 \mathrm{~K}$. This means, overall heating is much larger as BCS surface resistance increases exponentially with temperature. Nonetheless, this should not affect hot spots location. Heating is spread more evenly along the azimuthal angle $\varphi$. We expected this, since the orientation of $\vec{B}$ with respect to the cavity wall is independent of $\varphi$. 


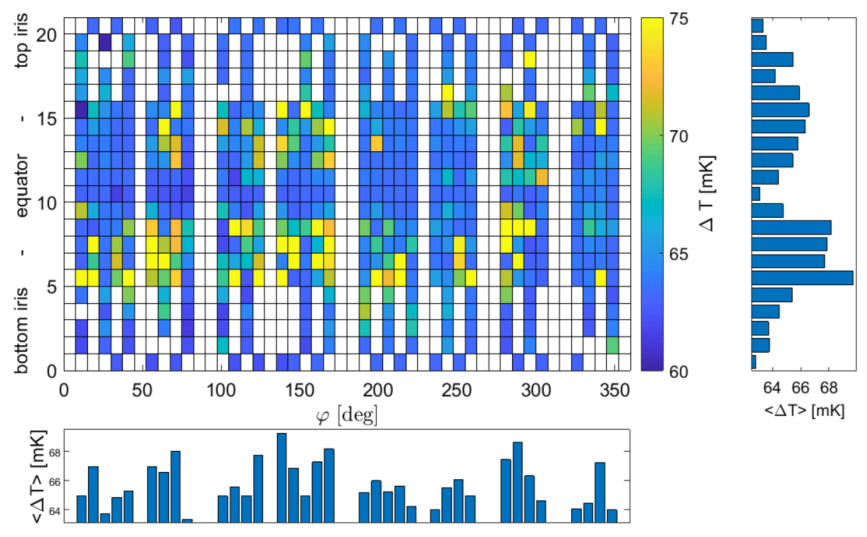

FIG. 12. Relative heating for applied field in $\alpha=90^{\circ}$. The data is recorded at $1.86 \mathrm{~K}$, and $E_{\text {acc }}=15 \mathrm{MW} / \mathrm{m}$. The quality factor is $Q_{0}=4.4 \times 10^{9}$.

The comparison of the row bar charts in Fig. 12 to those in Fig. 10, and 11 reveals that hot spots moved away from the equator region and toward the irises corresponding to where the surface and the B-field are perpendicular.

A top bottom asymmetry exists, with the bottom half of the cavity seemingly heating up more than the top half. This is likely caused by cooling dynamics: The cavity is cooled from the bottom up, therefore, the niobium becomes superconducting at the bottom first. While the phasefront moves to the top, some flux is expelled and, presumably alters the flux density further above. In [18] similar observations are recorded. When cooling down at a fast, or at a medium speed, an asymmetry is visible, whereas, during a slow cooldown no asymmetry is visible. In the case of partial trapping during fast, or medium cooldowns, the bottom half of the cavity becomes superconducting first. Thereby, expelling flux that influences the flux density during phase transition in the top half of the cavity. Hence, frozen flux is distributed differently across the cavity.

This observation supports the conclusion that a more sophisticated trapped flux model is needed.

Temperature maps can also be used to calculate local surface resistance. This is done as follows: The quality factor of the baseline measurements is used to calculate average surface resistance, $\left\langle R_{\mathrm{s}}\right\rangle$, of the cavity without applied field using $R_{\mathrm{s}}=\frac{G}{Q}$, where $Q$ is the quality factor, and $G$ is the geometry factor of a TESLA single cell cavity with $G=270 \Omega$. Assuming a homogeneous surface resistance allows to calibrate the thermal connection of the sensors.

Rise in temperature $\Delta T$ is proportional to surface resistance when the rf field is turned on. It is also proportional to the square of the $\mathrm{rf}$ magnetic field $H_{\mathrm{rf}}^{2}$. For every sensor a calibration constant $\xi_{i}$ is introduced, so the measured temperature rise of the respective sensor $\Delta T_{i}$ corresponds to the mean surface resistance $\left\langle R_{\mathrm{S}}\right\rangle$.

$$
\begin{gathered}
\left\langle R_{\mathrm{s}}\right\rangle=\frac{\xi_{i} \Delta T_{i}}{H_{\mathrm{rf}}^{2}} \\
\Leftrightarrow \xi_{i}=\frac{\left\langle R_{\mathrm{s}}\right\rangle H_{\mathrm{rf}}^{2}}{\Delta T_{i}}=\frac{G H_{\mathrm{rf}}^{2}}{Q_{0} \Delta T_{i}}
\end{gathered}
$$

The surface resistance is not perfectly homogeneous across the cavity, even without magnetic field applied during cooldown. Nonetheless, compared to the differences observed when an external field is applied, this temperature fluctuation is an order of magnitude smaller.

In subsequent calculations, only the middle part (row 5 to 15 ) of the temperature maps are taken into account. This is done due to more consistent coverage of the surface with temperature sensors, and only $5 \%$ deviation in the rf magnetic field across this area. Since the thermal contact of the sensors varies significantly, temperature points are averaged with the four adjacent points by weighing the point itself times four and the surrounding sensors times one. If the sensor is on an edge, it is averaged with the remaining nearest neighbors. This gives a more even, and realistic temperature map.

In a first step, the calibration constant $\xi_{i}$ is determined for every sensor without any applied external field, according to Eq. (5). In order to obtain the actual surface resistance maps, $\Delta T_{i}$ is measured with applied magnetic field during phase transition. This enables to calculate local surface $R_{\mathrm{s} i}$ according to:

$$
R_{\mathrm{s} i}=\frac{\xi_{i} \Delta T_{i}}{H_{r f}^{2}} .
$$

Figure 13 shows the calculated surface resistance when the field is applied in $\varphi=0^{\circ}, \alpha=0^{\circ}$.

By introducing the calibration constant $\xi_{i}$ and by averaging the data points with their nearest neighbors, the surface resistance's dependency on $\varphi$ becomes clearer. Figure 14

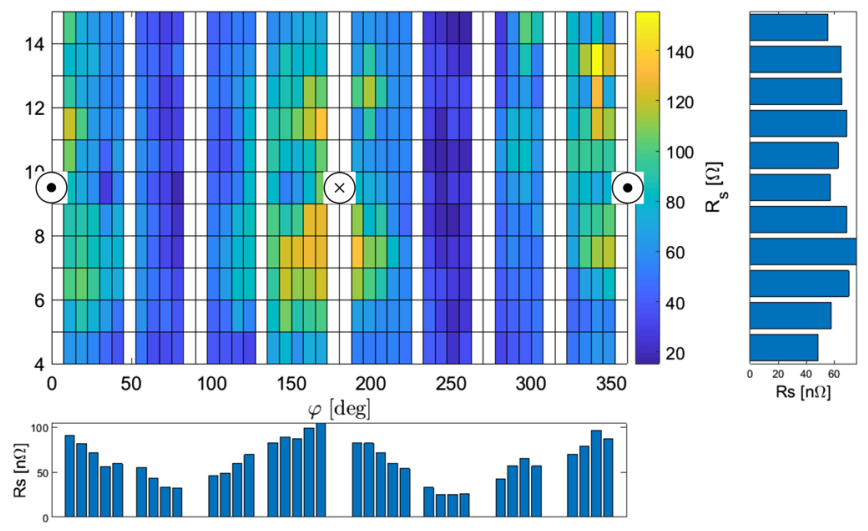

FIG. 13. Surface resistance inferred from $\Delta T$ measurements with applied field in $\varphi=0^{\circ}, \alpha=0^{\circ}$. 


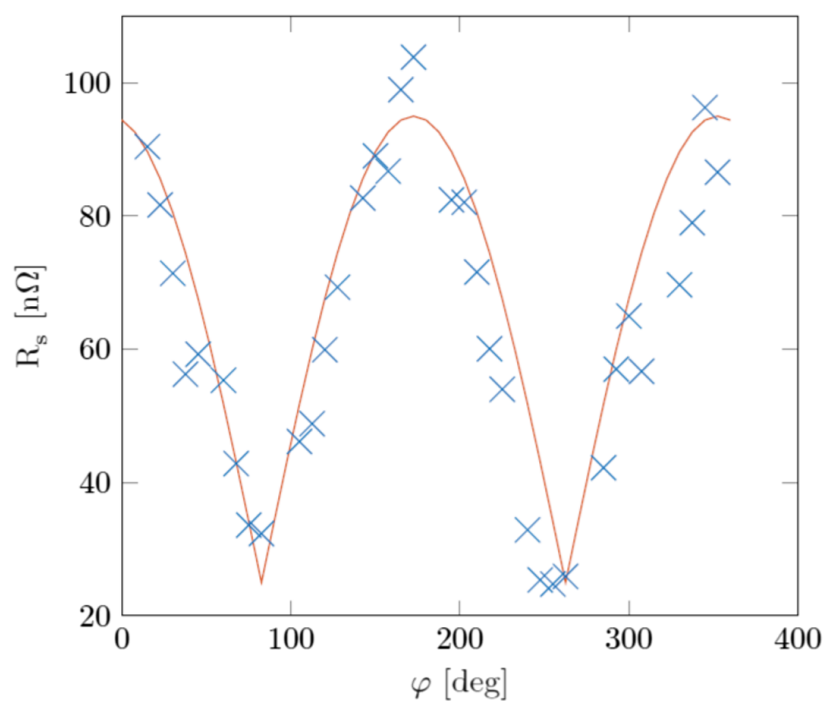

FIG. 14. Calculated surface resistance vs azimuthal angle, with applied field in $\varphi=0^{\circ}, \alpha=0^{\circ}$. Blue marks show averages of columns in Fig. 13. The red line follows $y=(70 \times \mid \cos (\varphi+$ $7.5) \mid+25) \mathrm{n} \Omega$.

shows surface resistance versus azimuthal angle $\varphi$. Since the B-field perpendicular to the surface is suspected to cause the increase in surface resistance, $|\cos (\varphi)|$ is included in the plot. Absolute values are used because for surface resistance $R_{\mathrm{S}}$ it is irrelevant whether frozen flux points into or out of the surface. The cosine is shifted $7.5^{\circ}$ to the left, as the field is perpendicular at $352.5^{\circ}$ and $172.5^{\circ}$ instead of $0^{\circ}$ and $180^{\circ}$. Furthermore, it is scaled up, and shifted upwards to fit the data points. The resulting equation is $y=(70 \times|\cos (\varphi+7.5)|+25) \mathrm{n} \Omega$. The $|\cos (\varphi)|$ dependency is also observed in [19].

Figure 14 supports the conclusion that the B-field perpendicular to the surface creates the largest contribution to surface resistance. The quality factor without applied field is $2.7 \times 10^{10}$. This corresponds to a surface resistance of $10 \mathrm{n} \Omega$. In Fig. 14, the residual resistance drops merely to $23 \mathrm{n} \Omega$. For angles where the field is parallel to the surface, we observe that the surface resistance is significantly lower compared to perpendicular field, yet not as low as in the absence of any applied field. This potentially indicates that a parallel component increases surface resistance as well but further tests are required to confirm this conclusion, since temperature differences of $1 \mathrm{mK}$ correspond to an increase of $20 \mathrm{n} \Omega$ for average sensors. With the data set at hand, it is not possible to give a quantitative result, of how much flux parallel or perpendicular to the surface increase surface resistance: While the increase in surface resistance in $\varphi=90^{\circ}, \alpha=0^{\circ}$ is consistent with the increase reported above, the increase for applied field in $\alpha=90^{\circ}$ is different, as described below.

Qualitatively plots in Figs. 15, and 16 showing calculated surface resistance for applied field in $\alpha=90^{\circ}$ do not

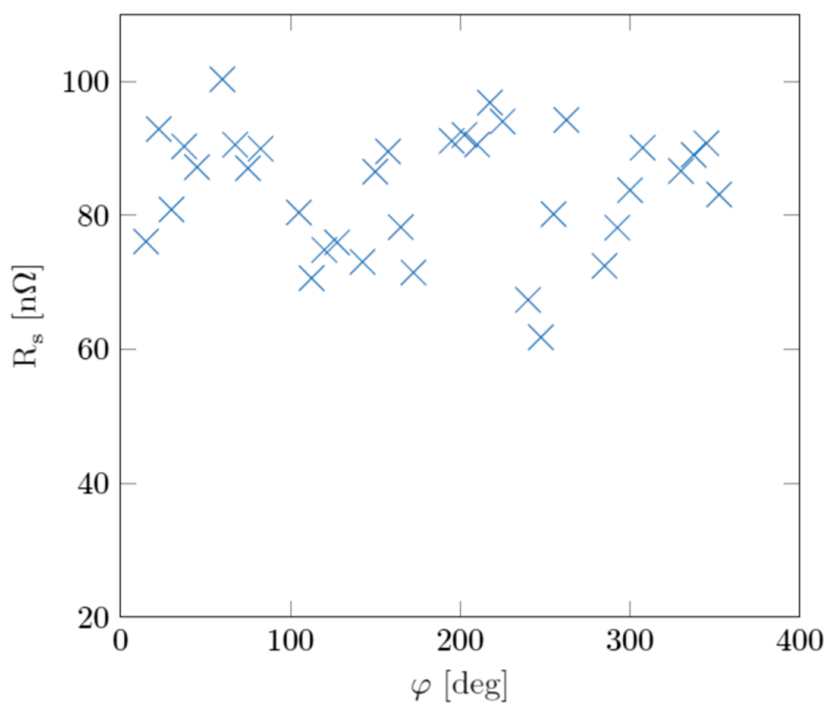

FIG. 15. Calculated surface resistance vs azimuthal angle, with applied field in $\alpha=90^{\circ}$. The surface resistance is mapped similarly to Fig. 13. Blue marks show average surface resistance of each column in the corresponding map. The map is not depicted.

differ from the expected result: There is no dependency of surface resistance on the azimuthal angle. The dependency on the polar angle reveals that surface resistance is minimal at the equator where the field is parallel to the surface. Moreover, the top bottom asymmetry reported earlier is visible. But the increase in surface resistance for the case of applied field in $\alpha=90^{\circ}$ is consistent with, but of different magnitude than, the cases of applied field in $\varphi=0^{\circ}$, or $\varphi=90^{\circ}$, and $\alpha=0^{\circ}$.

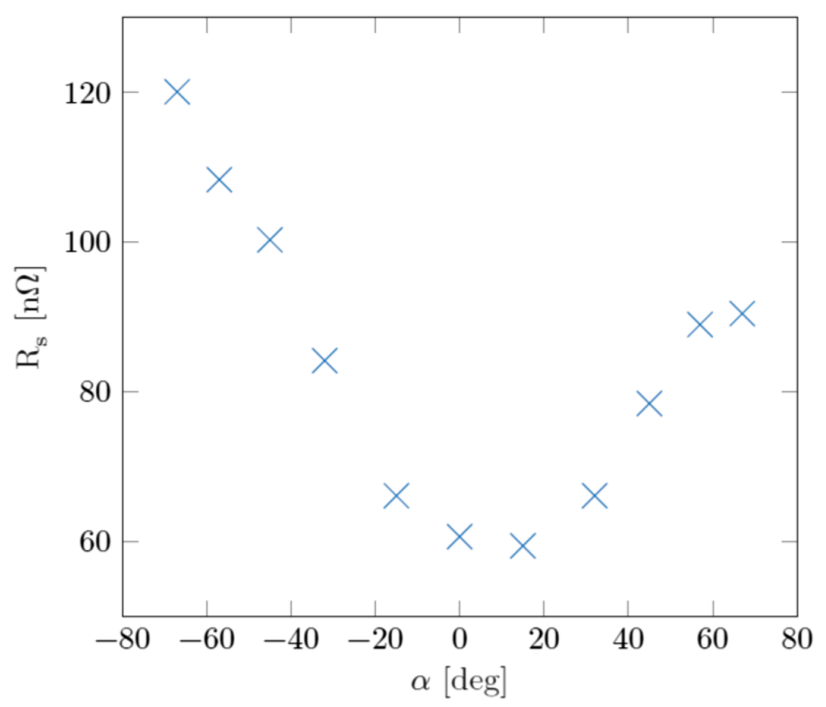

FIG. 16. Calculated surface resistance vs polar angle, with applied field in $\alpha=90^{\circ}$. The surface resistance is mapped similarly to Fig. 13. Blue marks show average surface resistance of each row in the corresponding map. The map is not depicted. 


\section{SUMMARY AND OUTLOOK}

\section{A. Conclusions}

Using the three dimensional magnetic field mapping system, it is possible to resolve, and visualize the magnetic field surrounding a superconducting cavity for the first time. Both, the magnetic flux mapping, and the temperature mapping measurements where a top bottom asymmetry is observed, show that the flux trapping mechanism cannot be fully explained by a simple static model. Therefore, the estimation of the amount of trapped flux using a few field probes can be a useful tool, but it has limits. Hence, our findings support the influence of partial trapping for the top bottom asymmetry proposed in [18].

Q vs E measurements for each applied magnetic field at different temperatures allowed for extraction of the residual resistance. There is a clear dependency on the angle of the applied field. The temperature maps suggest that the increase in residual resistance is strongest where the B-field is perpendicular to the surface. The percentage of the B-field perpendicular to the surface is calculated, and shows a similar trend to the residual resistance.

\section{B. Outlook}

We are currently developing more sophisticated simulation models which also implement dynamical effects in order to obtain a better fit with the measured data.

[1] H. Padamsee, J. Knobloch, and T. Hays, RF Superconductivity for Accelerators, 1st ed. (Wiley, New York, 1998).

[2] J. Bardeen, L. N. Cooper, and J. R. Schrieffer, Theory of superconductivity, Phys. Rev. 108, 1175 (1957).

[3] A. Gurevich, Theory of RF superconductivity for resonant cavities, Supercond. Sci. Technol. 30, 034004 (2017).

[4] C. Vallet, M. Bolore, B. Bonin, J. Charrier, B. Daillant, J. Gratadour, F. Koechlin, and H. Safa, Flux trapping in superconducting cavities, Proceedings of the 3. European particle accelerator conference. V. 2 (1992), pp. 1295-1297, https://accelconf.web.cern.ch/e92/PDF/ EPAC1992_1295.PDF.

[5] A. Gurevich and G. Ciovati, Effect of vortex hotspots on the radio-frequency surface resistance of superconductors, Phys. Rev. B 87, 054502 (2013).

[6] A. Gurevich and G. Ciovati, Dynamics of vortex penetration, jumpwise instabilities, and nonlinear surface resistance of
type-II superconductors in strong rf fields, Phys. Rev. B 77, 104501 (2008).

[7] History provided by DESY.

[8] B. Schmitz, J. Köszegi, K. Alomari, O. Kugeler, and J. Knobloch, Magnetometric mapping of superconducting RF cavities, Rev. Sci. Instrum. 89, 054706 (2018).

[9] B. Schmitz, Ortsauflösende Magnetometrie zur Untersuchung von eingefrorenem Magnetfluss in supraleitenden Kavitäten, Master's thesis, Universtät Siegen, 2017.

[10] AFF755B MagnetoResistive Field Sensor, https:// www.sensitec.com/fileadmin/sensitec/Service_and_Support/ Downloads/Data_Sheets/AFF700_800/SENSITEC_ AFF755B_DSE_06.pdf (2018).

[11] B. Schmitz, O. Kugeler, J. Köszegi, and K. Alomari, Patent No DE 102017127578 A1 2019.05.23: Vorrichtung und Verfahren zur Nutzung eines anisotrop magnetoresistiven Sensors zur Bestimmung von Magnetfeldern und Temperaturen.

[12] J. Knobloch, H. Muller, and H. Padamsee, Design of a high speed, high resolution thermometry system for $1.5 \mathrm{GHz}$ superconducting radio frequency cavities, Rev. Sci. Instrum. 65, 3521 (1994).

[13] F. Kramer, Mapping trapped flux and the related surface resistance in superconducting cavities, Master's thesis, Universtät Siegen, 2019.

[14] COMSOL, https://www.comsol.com (2019).

[15] A. Romanenko, A. Grassellino, O. Melnychuk, and D. A. Sergatskov, Dependence of the residual surface resistance of superconducting radio frequency cavities on the cooling dynamics around tc, J. Appl. Phys. 115, 184903 (2014), https://doi.org/10.1063/1.4875655.

[16] S. Huang, T. Kubo, and R. L. Geng, Dependence of trapped-flux-induced surface resistance of a large-grain $\mathrm{nb}$ superconducting radio-frequency cavity on spatial temperature gradient during cooldown through $T_{c}$, Phys. Rev. Accel. Beams 19, 082001 (2016).

[17] P. Dhakal, G. Ciovati, and A. Gurevich, Flux expulsion in niobium superconducting radio-frequency cavities of different purity and essential contributions to the flux sensitivity, Phys. Rev. Accel. Beams 23, 023102 (2020).

[18] S. Posen, M. Checchin, C. Crawford, A. Grassellino, M. Martinello, O. Melnychuk, A. Romanenko, D. Sergatskov, Z. Sung, and Y. Trenikhina, Fundamental studies of flux trapping, TESLA Technology Collaboration (TTC) Meeting (2018).

[19] R. Romijn, W. Weingarten, and H. Piel, Calibration of the scanning thermometer resistor system for a superconducting accelerating cavity, IEEE Trans. Magn. 19, 1318 (1983). 